\title{
Avances en Inmunidad Gestacional
}

\author{
Advances in Gestational Immunity
}

\author{
*M. E. Arias \& **** J. Villegas
}

\begin{abstract}
ARIAS, M. E. \& VILLEGAS, J. Avances en inmunidad gestacional. Int. J. Morphol., 28(3):713-718, 2010.
RESUMEN: El inicio y establecimiento de la gestación en los mamíferos dependen de la adaptación del sistema inmunológico de la madre para tolerar un feto semi-alogénico. La gestación en sí misma constituye un acontecimiento de equilibrio inmunológico, ya que mientras el sistema inmune mantiene la competencia para la defensa contra antígenos foráneos, mecanismos de tolerancia local y periférica previenen una respuesta inapropiada contra alo-antígenos fetales de origen paterno lo que pudiera provocar el rechazo del feto. La interacción materno-fetal es extremadamente compleja y es difícil determinar todos los componentes del sistema inmune involucrados. Hasta ahora se ha demostrado la participación activa de las células $\mathrm{T}$ y sus productos, las citoquinas y también se ha involucrado a las moléculas del complejo mayor de histocompatibilidad, los antígenos paternos y algunos inmunomoduladores como progesterona, indoleamina 2,3-dioxigeneasa y glicodelina, entre otros. Todos estos elementos parecen confluir para producir un gran cambio sistémico en el sistema inmune materno, promoviendo por una parte la tolerancia materno-fetal, crucial para finalmente permitir una gestación exitosa y, por otro lado, manteniendo una activa vigilancia inmune contra las infecciones que pondrían en riesgo la gestación y sobrevivencia de diversas especies. Se revisó la literatura más reciente acerca de los diferentes componentes del sistema inmune que han demostrado ser clave en el inicio y mantención de la gestación en mamíferos.
\end{abstract}

PALABRAS CLAVE: Embarazo/preñez; Tolerancia inmunológica; Inmunología de la reproducción.

\section{INTRODUCCIÓN}

El embarazo constituye una paradoja desde un punto de vista inmunológico ya que los mecanismos normales de protección contra las infecciones son potencialmente capaces de rechazar y destruir al feto debido a que éste puede ser visto por el sistema inmune materno como un injerto semi-alogénico. Sin embargo, un amplio repertorio de estrategias de evasión del sistema inmune contribuye a la sobrevivencia del feto durante la gestación. Entre dichas estrategias de evasión se encuentra el silenciamiento o modulación de los mecanismos de inducción en los compartimientos inmunes innato y adaptativo. El estudio de los diferentes componentes del sistema inmune han buscado una explicación para el inicio y establecimiento de una gestación exitosa, la cual se caracteriza porque la madre conserva su capacidad de respuesta inmunitaria al mismo tiempo que el feto es objeto de una protección muy especial.

Se realizó una revisión de la literatura más reciente acerca de los diferentes componentes del sistema inmune, cuya participación es clave tanto en el inicio como en la mantención de la gestación en mamíferos.

Inmunidad mediada por células T. Entre los diferentes mecanismos que se han propuesto hasta ahora para explicar la tolerancia inmunológica hacia embriones semi-alogénicos, se encuentran los mediados por anticuerpos antifosfolípidos, células NK, moléculas HLA-G solubles y el balance de citoquinas Th1/Th2 de células T (Christiansen et al., 2006). Se ha afirmado que un desbalance entre estos componentes puede conducir a abortos en ratón y en humanos. Por una parte, ratones hembras preñadas presentan normalmente una elevada inmunidad tipo-Th2 y se ha afirmado que la presencia de una excesiva inmunidad tipo-Th1 puede inducir rechazo fetal y abortos (Wegmann et al., 1993). Por otra parte, también se ha descrito la presencia de un perfil exacerbado de citoquinas tipo-Th2 en mujeres con pérdida recurrente de embarazos (RPL), con respecto a embarazadas contro-

\footnotetext{
* Centro de Excelencia de Biotecnología de la Reproducción, Universidad de La Frontera, Temuco, Chile.

** Departamento Medicina Interna, Facultad de Medicina, Universidad de La Frontera, Temuco, Chile. Financiado por: DIUFRO DI09-1006, Dirección de Investigación, Universidad de La Frontera.
} 
les. Sin embargo, no se encontró diferencia en el perfil de citoquinas entre las pacientes RPL que lograron completar un embarazo con éxito y aquellas que tuvieron abortos. Además, el hallazgo de una elevada inmunidad tipo-Th1 luego de un diagnóstico de aborto (Bates et al., 2002), sugiere que la inmunidad tipo-Th1 podría ser un efecto más que una causa de aborto (Christiansen et al., 2005). Adicionalmente, se ha demostrado la expresión de prácticamente todas las citoquinas en la decidua, en las diferentes etapas de la gestación y al menos en ratón, las citoquinas Th1 no serían necesariamente perjudiciales. Algunas de ellas, como LIF, IL11 e IFNg, incluso parecen ser requeridas para la implantación de blastocistos en ratones hembras (revisado en Szekeres-Bartho et al., 2009).

Células T reguladoras. La gestación requiere adaptaciones en los sistemas inmunes maternos, innato y adaptativo, que permitan evitar el rechazo inmune del feto semialogénico (Trowsdale \& Betz, 2006). Dentro del sistema inmune adaptativo, hay evidencias indicando que los linfocitos maternos preparados para reconocer y reaccionar con antígenos, como las moléculas del complejo mayor de histocompatibilidad y otras moléculas de origen paterno expresadas en el feto (Tafuri et al., 1995). Por ello, para evitar la respuesta de los linfocitos citotóxicos que causarían daño al tejido embrionario, debe generarse un estado activo de tolerancia inmune (Robertson et al., 2009). Esta tolerancia es mediada en parte por células $\mathrm{T}$ reguladoras (Treg), una subpoblación única de células T CD4+CD25+, que son potentes supresores de la generación y función efectora de la respuesta inmune inflamatoria mediada celularmente (Aluvihare et al., 2004).

El requerimiento fisiológico de células Treg para la gestación exitosa se demostró por primera vez a través de experimentos, en los que poblaciones de células T completas o depletadas de células Treg fueron transferidas a ratones hembras preñadas deficientes de células T. En ausencia de células Treg se observó rechazo y, por el contrario, las hembras transferidas con células T incluyendo células Treg, mostraron aceptación de los fetos alogénicos (Aluvihare $e t$ $a l$. .). En la decidua de hembras propensas a sufrir abortos espontáneos CBA/J x DBA/2J, se ha observado baja cantidad de células Treg y elevada actividad de células Th1 (Zenclussen et al., 2005), sugiriendo que las células Treg son esenciales para suprimir la inmunidad destructiva durante la gestación. Los eventos por los cuales las células Treg se activan y expanden para favorecer el embarazo todavía no están bien definidos (Aluvihare et al.). Se ha afirmado que las células Treg generadas en la periferia deben experimentar procesos de activación conducidos por antígenos para ejercer completamente su función supresora (Samy et al., 2006). Sin embargo, también se ha afirmado que las células Treg se expanden sistémicamente, y que la presencia de aloantígenos fetales no es necesaria para la expansión de las células Treg dentro del compartimento linfoide secundario durante la preñez (Aluvihare et al.). No obstante, la expresión de aloantígenos fetales conduce a un incremento del número de células Treg y de la supresión específica de la reactividad de los anticuerpos antialoantígenos paternos (Zhao et al., 2007). En mujeres se han encontrado incrementos similares en las células Tregs sistémicas y deciduales. Las células CD4+CD25+ se incrementan tempranamente en el embarazo, alcanzando un máximo en el segundo trimestre y descendiendo después del parto (Somerset et al., 2004).

Células natural killer. El útero de hembras gestantes contiene un elevado número de leucocitos especializados que trabajan coordinadamente para controlar el desarrollo e invasión del trofoblasto, el desarrollo de la angiogénesis y también regular la inmunidad local (Ashkar et al., 2000). El análisis de las células inmunes presentes en la interfase materno-fetal en ratones y humanos, muestra que la población predominante corresponde a células natural killer uterinas (uNK) (Karsten et al., 2009). Las células uNK producen elevadas concentraciones de factores de crecimiento angiogénicos, los que son muy importantes en los procesos de placentación e implantación (Saito et al., 2008), mantienen la integridad de la decidua y protegen al feto contra patógenos externos (Ashkar et al.), por lo que son cruciales para el éxito de la gestación. Los precursores de las células uNK parecen derivar principalmente del tejido linfoide cercano al útero (Karsten et al.). Las células NK se clasifican de acuerdo a sus marcadores de superficie como células NK CD16+CD56dim y células NK CD16-CD56bright (MoffettKing, 2002). En sangre periférica, la principal población de células NK son las células NK CD16+CD56dim, representando el 10-15\% de los linfocitos, mientras las células NK CD16+CD56bright son una población menor que representa aproximadamente un $0.5 \%$. En cambio, en el endometrio, las células NK CD16-CD56bright constituyen la principal población que representa un $20 \%$ de los linfocitos del endometrio en fase proliferativa, incrementa a 40-50\% en la fase secretora y alcanza hasta un $70-80 \%$ en la decidua en gestación temprana (Saito et al., 2008).

Las células NK presentes en la decidua tienen además una función fundamental en la mantención de la gestación. Las células uNK poseen un receptor que reconoce moléculas CMH de clase I no polimórficas, HLA-C, HLAG y HLA-E. Dichas moléculas son expresadas en el trofoblasto extravelloso y su reconocimiento por parte de las células uNK impide su activación y con ello el ataque al feto. Sin embargo, se ha observado que células uNK que no expresan el receptor inhibitorio, son menos sensibles a las 
señales enviadas por sus receptores de activación, manteniendo igualmente la tolerancia al feto durante la preñez (MacDonald, 2005). Adicionalmente, las células NK de la decidua pueden promover tolerancia gestacional destruyendo las células dendríticas del feto, que al migrar hacia la decidua activan directamente a las células T maternas e inducen rechazo fetal (Saito et al., 2008).

Moléculas del complejo mayor de histocompatibilidad. El sinciotrofoblasto tiene contacto directo con los linfocitos maternos circulantes en el espacio intervelloso. Resulta interesante que, el sinciotrofoblasto no exprese antígenos del complejo mayor de histocompatibilidad (CMH). Por otra parte, el trofoblasto extravellositario y la decidua expresan las moléculas CMH de clase I menos polimórficas, HLA-C, HLA-G, HLA-E y HLA-F, y no expresan antígenos CMH de clase II. Bajo tales circunstancias, las células inmunocompetentes maternas no tienen contacto efectivo con células expresando alo-antígenos paternos, por lo que durante largo tiempo se ha creído que en gestaciones alogénicas no sería necesario desarrollar tolerancia específica a antígenos paternos (Saito et al., 2007). Posteriormente han surgido dudas sobre la presencia de tolerancia específica a moléculas CMH clase I o clase II durante la gestación humana (Moffett-King, 2002). Estudios recientes han demostrado que la placenta no es una barrera física impenetrable y no previene el intercambio de células entre la madre y el feto (Saito et al., 2007). Al contrario, el intercambio celular entre madre y feto es común y se pueden detectar células fetales en la circulación materna y también células maternas en los recién nacidos por largo tiempo (Adams et al., 2007). Además, una gran cantidad de desechos celulares del trofoblasto se liberan hacia la circulación materna (Huppertz et al., 2003). Estos desechos celulares en su mayoría derivan de células senescentes y en apoptosis (Saito et al., 2007). Datos recientes señalan que antígenos del CMH clase II tales como HLA-DR y HLA-DQ, pero no HLA$\mathrm{DM}$, se expresan sobre el retículo endoplásmico de las células descartadas del trofoblasto (Ranella et al., 2005). Cuando las células dendríticas incorporan antígenos $\mathrm{CMH}$ clases I y II que se expresan sobre células del trofoblasto de desecho, presentan estos antígenos a células T CD8+ y a células T CD4+. Por este mecanismo, antígenos CMH de clase II presentados a las células T CD4+ pueden estimular su diferenciación a células Treg en la periferia y los antígenos $\mathrm{CMH}$ de clase I pueden estimular la activación de células T CD8+ maternas. Sin embargo, la activación de células CD8+ está bajo control supresor por el sistema Fas/FasL, y por el sistema de moléculas solubles HLA-G1, presente en la interfase materno-fetal. También se ha observado que la fagocitosis de células fetales apoptóticas por parte de células dendríticas y macrófagos resulta en una activa inmunosupresión y respuesta anti-inflamatoria (Saito et al., 2007).
Antígenos paternos. El sistema inmune femenino se expone por primera vez a los antígenos paternos durante el coito, cuando el fluido seminal se libera en el tracto reproductivo (Robertson, 2005). Ello abre la posibilidad de que el sistema inmune de las hembras pueda reconocer y responder a los antígenos del macho presentes en el líquido seminal y junto con ello, de que dichos antígenos puedan activar la respuesta inmune en un embarazo posterior (Robertson et al., 2009). Los tejidos reproductivos de la hembra se exponen a antígenos paternos durante la gestación durante dos etapas diferentes, primero cuando se introduce el fluido seminal durante el apareamiento y luego, cuando las células del trofoblasto invaden el tejido materno después de la implantación (Robertson \& Sharkey, 2001). Moldenhauer et al. (2009) han propuesto que las células T de la hembra reconocen y responden a los antígenos presentes en el fluido seminal y que la exposición a tales antígenos en el período de la concepción es el primer evento en la respuesta inmune materna durante la gestación. Adicionalmente, factores solubles del plasma seminal inducen la expresión de citoquinas pro-inflamatorias y quimioquinas en las células epiteliales del cérvix y útero, lo que tiene como consecuencia la acumulación de células dendríticas, macrófagos y granulocitos en el estroma y lumen endometriales (Robertson et al., 1996). Una respuesta inmune contra antígenos seminales podría tener efectos adversos sobre la viabilidad de los espermatozoides, en la fecundación de ovocitos y ser incompatible con la mantención de la gestación (Shetty et al., 2008), ya que el feto exhibe moléculas del CMH y otros antígenos paternos (Thaler, 1989). No obstante lo anterior, el semen es también una fuente rica de agentes que modulan la respuesta inmune y que son conocidos como potentes inductores de las células Treg (Moldenhauer et al.).

Inmunomoduladores. Tradicionalmente, se ha considerado al útero gestante como un lugar inmunológicamente privilegiado, donde el feto es protegido del rechazo por el sistema inmune materno. En esta protección participan varias moléculas con acción inmunomoduladora, entre las que encontramos a progesterona, glicodelina y la enzima indoleamina 2,3-dioxigenasa.

Progesterona. La progesterona es fundamental para establecer y mantener la gestación, ya que regula el sangrado durante la menstruación, repara y regenera tejidos, participa en la inflamación, angiogénesis y al final del embarazo interfiere con el metabolismo del ácido araquidónico contribuyendo de esta manera al estado de reposo del útero (Szekeres-Bartho et al.). Además de sus efectos sobre el útero, la progesterona interactúa con los sistemas inmunológico y endocrino creando un medio ambiente inmunológico favorable para el feto. Se ha demostrado que la progesterona bloquea la proliferación de linfocitos estimulados por 
mitógenos, reduce la producción de citoquinas proinflamatorias en respuesta a antígenos bacterianos en macrófagos, prolonga la sobrevida de los injertos, regula la producción de anticuerpos, disminuye el estallido oxidativo en los monocitos y actúa sobre el perfil de citoquinas durante el embarazo (Beagley \& Gockel, 2003).

Las células dendríticas del útero pueden inducir tolerancia frente a antígenos o al revés, pueden iniciar una fuerte respuesta inmune dependiendo de su estado de maduración o diferenciación al momento de la presentación del antígeno (Kammerer et al., 2000). La progesterona favorece la inducción de células dendríticas semi-maduras tolerogénicas, e inhibe la actividad de células dendríticas maduras que pueden generar respuestas proinflamatorias (Liang et al., 2006).

En el útero, la progesterona promueve la interacción de las células NK con el endotelio, favoreciendo de esta manera la creación de un entorno adecuado para el establecimiento de la gestación, y también estimula la expresión de mRNA de las quimiocinas CXCL10/IP-10, CX3CL1/ Fractalquina y CCL2/MCP-1 en células del estroma, resultando en un aumento de la capacidad migratoria de las células NK periféricas durante la preñez (Szekeres-Bartho et al.).

Glicodelina. La glicodelina, llamada también glicoproteína dimérica (GdA), es sintetizada por el endometrio bajo regulación de progesterona. La GdA pertenece a la familia de lipocalinas y es la glicoproteína más abundante del útero de primates durante la implantación y en las etapas tempranas de la gestación (Seppala et al., 2002). La GdA suprime la proliferación de las células B y células $\mathrm{T}$, inhibe la quimiotaxis de monocitos, la actividad de las células NK y regula diferencialmente la respuesta Th1/Th2 (Alok \& Karande, 2009). La actividad inhibitoria de GdA sobre las células $\mathrm{T}$ se realiza vía inducción de una cascada apoptótica (Mukhopadhyay et al., 2001). En el caso de monocitos y células NK, el efecto inhibitorio también se debe a la apoptosis inducida por GdA, aunque los eventos de señalización que conducen a la apoptosis parecen ser diferentes. La actividad y proliferación de las células B también se inhibe por la GdA; sin embargo, a diferencia de las otras células del sistema inmunológico, las células B no experimentan muerte celular por apoptosis o necrosis luego de ser tratadas con GdA (Alok \& Karande).

Indoleamina 2,3-dioxigeneasa. La enzima indoleamina 2,3dioxigeneasa (IDO) es uno de los principales actores involucrados en la inhibición de la proliferación celular, incluyendo la de las células $\mathrm{T}$ activadas. Recientemente, se ha demostrado un papel importante de la IDO en la inducción de tolerancia inmune durante la infección, embarazo, trasplante, autoinmunidad y neoplasia (Curti et al., 2009). IDO se expresa en células dendríticas, macrófagos, trofoblasto del ratón y trofoblasto velloso y extravelloso en humanos (Kudo et al., 2004). Datos recientes, señalan que la molécula inhibitoria CTL-4, la cual se expresa sobre células Treg, incrementa la actividad de IDO en células dendríticas y macrófagos para favorecer una gestación exitosa (Saito et al., 2007).

\section{CONCLUSIÓN}

La literatura más reciente en torno a la inmunología del embarazo indica que se necesitan diferentes componentes del sistema inmune, actuando en conjunto, para suprimir la respuesta inmune materna contra los alo-antígenos fetales, y que dichos componentes son particularmente esenciales durante los primeros días de gestación, previo al período de implantación. Adicionalmente, la respuesta inmune que puede generarse frente a patógenos durante el embarazo, es importante para proteger tanto a la madre como al feto, lo que finalmente permitirá lograr una gestación exitosa.

ARIAS, M. E. \& VILLEGAS, J. Advances in gestational immunity. Int. J. Morphol., 28(3):713-718, 2010.

SUMMARY: The initiation and establishment of pregnancy in mammals depends on the adaptation from maternal immune system to tolerate a semi-allogeneic fetus. Pregnancy itself constitutes an event of immune balance because, while the immune system maintains the capacity for defense against foreign antigens, mechanisms of local and peripheral tolerance may prevent an inappropriate response against fetal alloantigens of paternal origin which could lead to rejection of the fetus. The maternal-fetal immune interaction is extremely complex and it has therefore been difficult to identify all the immune components involved. So far, it is known that the active participation of T cells and their products, cytokines, and has also involved molecules from the major histocompatibility complex, other paternal antigens and some immunomodulators molecules such as progesterone, glycodelin and indoleamine 2,3-dioxigenase among others. All these elements seem to converge to produce a major systemic change in the maternal immune system, promoting on one hand the maternal-fetal tolerance, crucial to allow a successful pregnancy and on the other hand, maintaining an active immune surveillance against infections that might endanger pregnancy and survival of diverses species. A review of recent literature about the different components of the immune system that have proven key in the beginning and maintenance of pregnancy in mammals.

KEY WORDS: Pregnancy; Immune tolerance; Reproductive immunology. 


\section{REFERENCIAS BIBLIOGRÁFICAS}

Adams, K. M.; Yan, Z.; Stevens, A. M. \& Nelson, J. L. The changing maternal "self" hypothesis: A mechanism for maternal tolerance of the fetus. Placenta, 28:378-82, 2007.

Alok, A. \& Karande, A. A. The role of glycodelin as an immune-modulating agent at the feto-maternal interface. J. Reprod. Immunol., 83:124-7, 2009.

Aluvihare, V. R.; Kallikourdis, M. \& Betz, A. G. Regulatory $\mathrm{T}$ cells mediate maternal tolerance to the fetus. Nat. Immunol., 5:266-71, 2004.

Ashkar, A. A.; Di Santo, J. P. \& Croy, B. A. Interferon gamma contributes to initiation of uterine vascular modification, decidual integrity, and uterine natural killer cell maturation during normal murine pregnancy. J. Exp. Med., 192:259-70, 2000.

Bates, M. D.; Quenby, S.; Takakuwa, K.; Johnson, P. M. \& Vince, G. S. Aberrant cytokine production by peripheral blood mononuclear cells in recurrent pregnancy loss? Hum. Reprod., 17:2439-44, 2002.

Beagley, K. W. \& Gockel, C. M. Regulation of innate and adaptive immunity by the female sex hormones oestradiol and progesterone. FEMS Immunol. Med. Microbiol., 38:13-22, 2003.

Curti, A.; Trabanelli, S.; Salvestrini, V.; Baccarani, M. \& Lemoli, R. M. The role of indoleamine 2,3-dioxygenase in the induction of immune tolerance: Focus on hematology. Blood, 113:2394-401, 2009.

Christiansen, O. B.; Nielsen, H. S.; Kolte, A. \& Pedersen, A. T. Research methodology and epidemiology of relevance in recurrent pregnancy loss. Semin. Reprod. Med., 24:5-16, 2006.

Christiansen, O. B.; Nybo Andersen, A. M.; Bosch, E.; Daya, S.; Delves, P. J.; Hviid, T. V.; Kutteh, W. H.; Laird, S. M.; Li, T. C. \& van der Ven, K. Evidence-based investigations and treatments of recurrent pregnancy loss. Fertil. Steril., 83:821-39, 2005.

Huppertz, B.; Kingdom, J.; Caniggia, I.; Desoye, G.; Black, S.; Korr, H. \& Kaufmann, P. Hypoxia favours necrotic versus apoptotic shedding of placental syncytiotrophoblast into the maternal circulation. Placenta, 24:181-90, 2003.
Kammerer, U.; Schoppet, M.; McLellan, A. D.; Kapp, M.; Huppertz, H. I.; Kampgen, E. \& Dietl, J. Human decidua contains potent immunostimulatory CD83(+) dendritic cells. Am. J. Pathol., 157:159-69, 2000.

Karsten, C. M.; Behrends, J.; Wagner, A. K.; Fuchs, F.; Figge, J.; Schmudde, I.; Hellberg, L. \& Kruse, A. DC within the pregnant mouse uterus influence growth and functional properties of uterine NK cells. Eur. J. Immunol., 39:2203-14, 2009.

Kudo, Y.; Boyd, C. A.; Spyropoulou, I.; Redman, C. W.; Takikawa, O.; Katsuki, T.; Hara, T.; Ohama, K. \& Sargent, I. L. Indoleamine 2,3-dioxygenase: distribution and function in the developing human placenta. $J$. Reprod. Immunol., 61:87-98, 2004.

Liang, J.; Sun, L.; Wang, Q. \& Hou, Y. Progesterone regulates mouse dendritic cells differentiation and maturation. Int. Immunopharmacol., 6:830-8, 2006.

MacDonald, H. R. NK cell tolerance: Revisiting the central dogma. Nat. Immunol., 6:868-9, 2005.

Moffett-King, A. Natural killer cells and pregnancy. Nat. Rev. Immunol., 2:656-63, 2002.

Moldenhauer, L. M.; Diener, K. R.; Thring, D. M.; Brown, M. P.; Hayball, J. D. \& Robertson, S. A. Crosspresentation of male seminal fluid antigens elicits $\mathrm{T}$ cell activation to initiate the female immune response to pregnancy. J. Immunol., 182:8080-93, 2009.

Mukhopadhyay, D.; Sundereshan, S.; Rao, C. \& Karande, A. A. Placental protein 14 induces apoptosis in T cells but not in monocytes. J. Biol. Chem., 276:28268-73, 2001.

Ranella, A.; Vassiliadis, S.; Mastora, C.; Valentina, M.; Dionyssopoulou, E. \& Athanassakis, I. Constitutive intracellular expression of human leukocyte antigen (HLA)-DO and HLA-DR but not HLA-DM in trophoblast cells. Hum. Immunol., 66:43-55, 2005.

Robertson, S. A. Seminal plasma and male factor signalling in the female reproductive tract. Cell Tissue Res., 322: 43-52, 2005.

Robertson, S. A.; Guerin, L. R.; Moldenhauer, L. M. \& Hayball, J. D. Activating T regulatory cells for tolerance 
in early pregnancy - the contribution of seminal fluid. $J$. Reprod. Immunol., 83:109-16, 2009.

Robertson, S. A.; Mau, V. J.; Tremellen, K. P. \& Seamark, R. F. Role of high molecular weight seminal vesicle proteins in eliciting the uterine inflammatory response to semen in mice. J. Reprod. Fertil., 107:265-77, 1996.

Robertson, S. A. \& Sharkey, D. J. The role of semen in induction of maternal immune tolerance to pregnancy. Semin. Immunol., 13:243-54, 2001.

Saito, S.; Nakashima, A.; Myojo-Higuma, S. \& Shiozaki, A. The balance between cytotoxic NK cells and regulatory NK cells in human pregnancy. J. Reprod. Immunol., 77:14-22, 2008.

Saito, S.; Shima, T.; Nakashima, A.; Shiozaki, A.; Ito, M. \& Sasaki, Y. What is the role of regulatory T cells in the success of implantation and early pregnancy? J. Assist. Reprod. Genet., 24:379-86, 2007.

Samy, E. T.; Setiady, Y. Y.; Ohno, K.; Pramoonjago, P.; Sharp, C. \& Tung, K. S. The role of physiological self-antigen in the acquisition and maintenance of regulatory T-cell function. Immunol. Rev., 212:170-84, 2006.

Seppala, M.; Taylor, R. N.; Koistinen, H.; Koistinen, R. \& Milgrom, E. Glycodelin: A major lipocalin protein of the reproductive axis with diverse actions in cell recognition and differentiation. Endocr. Rev., 23:401-30, 2002.

Shetty, J.; Bronson, R. A. \& Herr, J. C. Human sperm protein encyclopedia and alloantigen index: Mining novel alloantigens using sera from ASA-positive infertile patients and vasectomized men. J. Reprod. Immunol., 77:23-31, 2008.

Somerset, D. A.; Zheng, Y.; Kilby, M. D.; Sansom, D. M. \& Drayson, M. T. Normal human pregnancy is associated with an elevation in the immune suppressive CD25+ CD4+ regulatory T-cell subset. Immunology, 112:38-43, 2004.

Szekeres-Bartho, J.; Halasz, M. \& Palkovics, T. Progesterone in pregnancy: receptor-ligand interaction and signaling pathways. J. Reprod. Immunol., 83:60-4, 2009.

Tafuri, A.; Alferink, J.; Moller, P.; Hammerling, G. J. \& Arnold, B. T cell awareness of paternal alloantigens during pregnancy. Science, 270:630-3, 1995.
Thaler, C. J. Immunological role for seminal plasma in insemination and pregnancy. Am. J. Reprod. Immunol., 21:147-50, 1989.

Trowsdale, J. \& Betz, A. G. Mother's little helpers: Mechanisms of maternal-fetal tolerance. Nat. Immunol., 7:241-6, 2006.

Wegmann, T. G.; Lin, H.; Guilbert, L. \& Mosmann, T. R. Bidirectional cytokine interactions in the maternal-fetal relationship: Is successful pregnancy a $\mathrm{TH} 2$ phenomenon? Immunol. Today, 14:353-6, 1993.

Zenclussen, A. C.; Gerlof, K.; Zenclussen, M. L.; Sollwedel, A.; Bertoja, A. Z.; Ritter, T.; Kotsch, K.; Leber, J. \& Volk, H. D. Abnormal T-cell reactivity against paternal antigens in spontaneous abortion: Adoptive transfer of pregnancy-induced $\mathrm{CD} 4+\mathrm{CD} 25+\mathrm{T}$ regulatory cells prevents fetal rejection in a murine abortion model. Am. J. Pathol., 166:811-22, 2005.

Zhao, J. X.; Zeng, Y. Y. \& Liu, Y. Fetal alloantigen is responsible for the expansion of the CD4(+)CD25(+) regulatory $\mathrm{T}$ cell pool during pregnancy. J. Reprod. Immunol., 75:71-81, 2007.

Dirección para correspondencia:

Dra. Juana Villegas

Centro de Excelencia de Biotecnología de la Reproducción

Universidad de La Frontera

Avenida Francisco Salazar 01145

Casilla 54-D

Temuco

CHILE

Fono: (56) 45325592

Fax: (56) 45325600

Email: jvillega@ufro.cl.

Recibido : 25-04-2010

Aceptado: $30-06-2010$ 